\title{
Laparoscopic radical cholecystectomy with common bile duct resection for T2 gallbladder cancer
}

\author{
Jonathan Geograpo Navarro ${ }^{1}$ and Chang Moo Kang ${ }^{2}$
}

\author{
${ }^{1}$ Division of Surgical Oncology, Department of Surgery, Vicente Sotto Memorial Medical Center, Cebu City, \\ Philippines, ${ }^{2}$ Division of Hepatobiliary and Pancreatic Surgery, Department of Surgery, Yonsei University College of \\ Medicine, ${ }^{3}$ Pancreatobiliary Cancer Center, Yonsei Cancer Center, Severance Hospital, Seoul, Korea
}

\begin{abstract}
The oncologic safety and feasibility of laparoscopic radical cholecystectomy for a preoperatively suspected gallbladder cancer is continually being challenged even in an era of minimally invasive surgery. A seventy-four-year-old woman was presented in the outpatient department with a history of fever, abdominal pain, and vomiting. CT scan showed an irregular wall thickening of the body to the cystic duct of the gallbladder and portocaval lymph node. In addition, EUS revealed no subserosal invasion of the tumor. PET scan showed an intense FDG uptake of in the gallbladder and in the portocaval lymph node. The laparoscopic radical cholecystectomy was performed with 6 trocars. The procedure included simple cholecystectomy, hepatoduodenal and aortocaval lymphadenectomy, and common bile duct resection. The hepaticojejunal anastomosis was constructed laparoscopically, while the jejunal continuity was established via an extracorporeal anastomosis. The patient was discharged on the 7th postoperative day with no complications and adjuvant chemotherapy was started on the 14th day after surgery. Based on our experienced, laparoscopic radical cholecystectomy with combined common bile duct resection is technically safe and feasible. (Ann Hepatobiliary Pancreat Surg 2019;23:69-73)
\end{abstract}

Key Words: Gallbladder cancer; Radical cholecystectomy; Common bile duct resection; Hepaticojejunostomy

\section{INTRODUCTION}

The role of laparoscopic surgery for the management of gallbladder cancer remains controversial. The current standard of care for preoperatively suspected gallbladder cancer is still an open radical cholecystectomy. ${ }^{1}$ In laparoscopic radical cholecystectomy, overcoming the innate complexity in the performance of adequate lymphadenectomy, liver resection, and possible common bile duct resection remains a challenge to surgeons. ${ }^{2}$

Recently, however, several high volume centers have demonstrated the efficacy and safety of laparoscopic radical cholecystectomy, which includes extended cholecystectomy and hepatoduodenal ligament lymphadenectomy in gallbladder cancer. ${ }^{3}$ Nevertheless, only a few reports on the feasibility of laparoscopic radical cholecystectomy with a common bile resection. ${ }^{4}$
With our expanding experience in minimally invasive surgery, our center has begun to employ the laparoscopic radical cholecystectomy in select patients with a preoperatively suspected early gallbladder cancer. We believe that as long as oncologic principles of open surgery have been carefully followed, laparoscopic surgery has a potential role in the management of early gallbladder cancer. Thus, we aim to report and describe our first successfully performed laparoscopic radical cholecystectomy with combined segmental resection of the bile duct in a patient with preoperatively suspected $\mathrm{T} 2$ gallbladder cancer near the cystic duct.

\section{CASE}

\section{Patient's clinical profile}

A 74-year-old woman with background history of hy-

Received: June 29, 2018; Revised: August 7, 2018; Accepted: August 18, 2018

Corresponding author: Chang Moo Kang

Division of Hepatobiliary and Pancreatic Surgery, Department of Surgery, Yonsei University College of Medicine, 50 Yonsei-ro, Seodaemun-gu, Seoul 03722, Korea

Tel: +82-2-2228-2100-3, Fax: +82-2-313-8289, E-mail: cmkang@yuhs.ac

Copyright (C) 2019 by The Korean Association of Hepato-Biliary-Pancreatic Surgery

This is an Open Access article distributed under the terms of the Creative Commons Attribution Non-Commercial License (http://creativecommons.org/ licenses/by-nc/4.0) which permits unrestricted non-commercial use, distribution, and reproduction in any medium, provided the original work is properly cited. Annals of Hepato-Biliary-Pancreatic Surgery • pISSN: 2508-5778 • elSSN: 2508-5859 
pertension presented in the outpatient department of Yonsei University Health System complaining of 2 weeks history of fever, abdominal pain and vomiting. She does not smoke or drink alcoholic beverages. Laboratory examinations revealed the following values: White blood count of $6.10103 / \mu 1$, alkaline phosphatase at $133 \mathrm{IU} / \mathrm{L}$ (47-147), aspartate aminotransferase 26 IU/L (13-34), alanine aminotransferase $9 \mathrm{IU} / \mathrm{L}$ (5-46), gamma-glutamyl transferase at $123(7.0-35.0)$, total bilirubin at $0.8 \mathrm{mg} / \mathrm{dL}$ (0.4-1.5), fasting blood glucose at $113 \mathrm{mg} / \mathrm{dL}$ (70-110), carcinoembryonic antigen at $2.73 \mathrm{ng} / \mathrm{ml}$, and cancer antigen $19-9$ at $140 \mathrm{U} / \mathrm{ml}(0.0-5.0)$.

The imaging from the computed tomography (CT) scan of the abdomen showed irregular wall thickening on the liver and peritoneal side of the gallbladder body with no definite liver invasion. The tumor also extended to the cystic duct. An enlarged lymph node at portocaval space, and a borderline sized lymph node at the hepatoduodenal ligament were also noted (Fig. 1A). Likewise, the endoscopic ultrasound (EUS) confirmed a liver side tumor in the body of the gallbladder with cystic duct involvement without serosal and liver invasion (Fig. 1B, C). On positron emission tomography (PET) scan, an intense fluorodeoxyglucose (FDG) uptake on the body of the gall- bladder and portocaval lymph node was noted, most likely a lymph node metastasis (Fig. 1D). Our preoperative impression was T2 gallbladder carcinoma with lymph node metastasis. Patient subsequently underwent laparoscopic radical cholecystectomy with common bile duct resection and Roux-en Y hepaticojejunostomy on January 12, 2018.

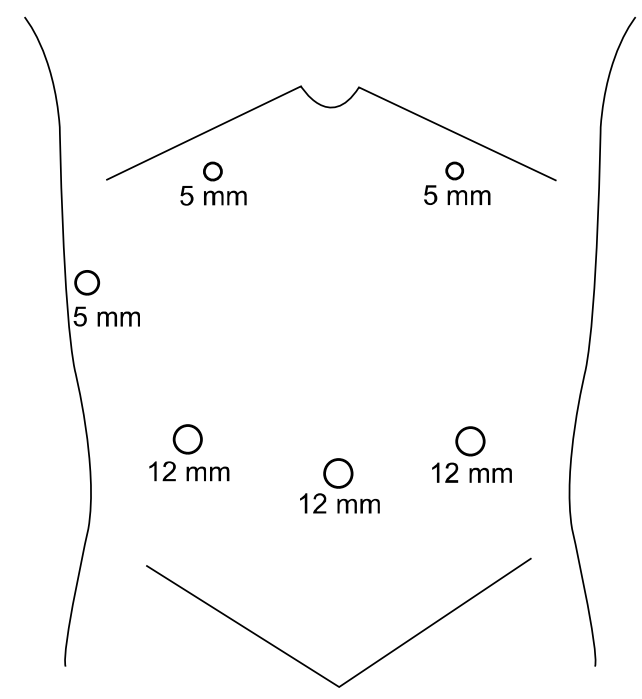

Fig. 2. Placement of trocars in laparoscopic radical cholecystectomy.
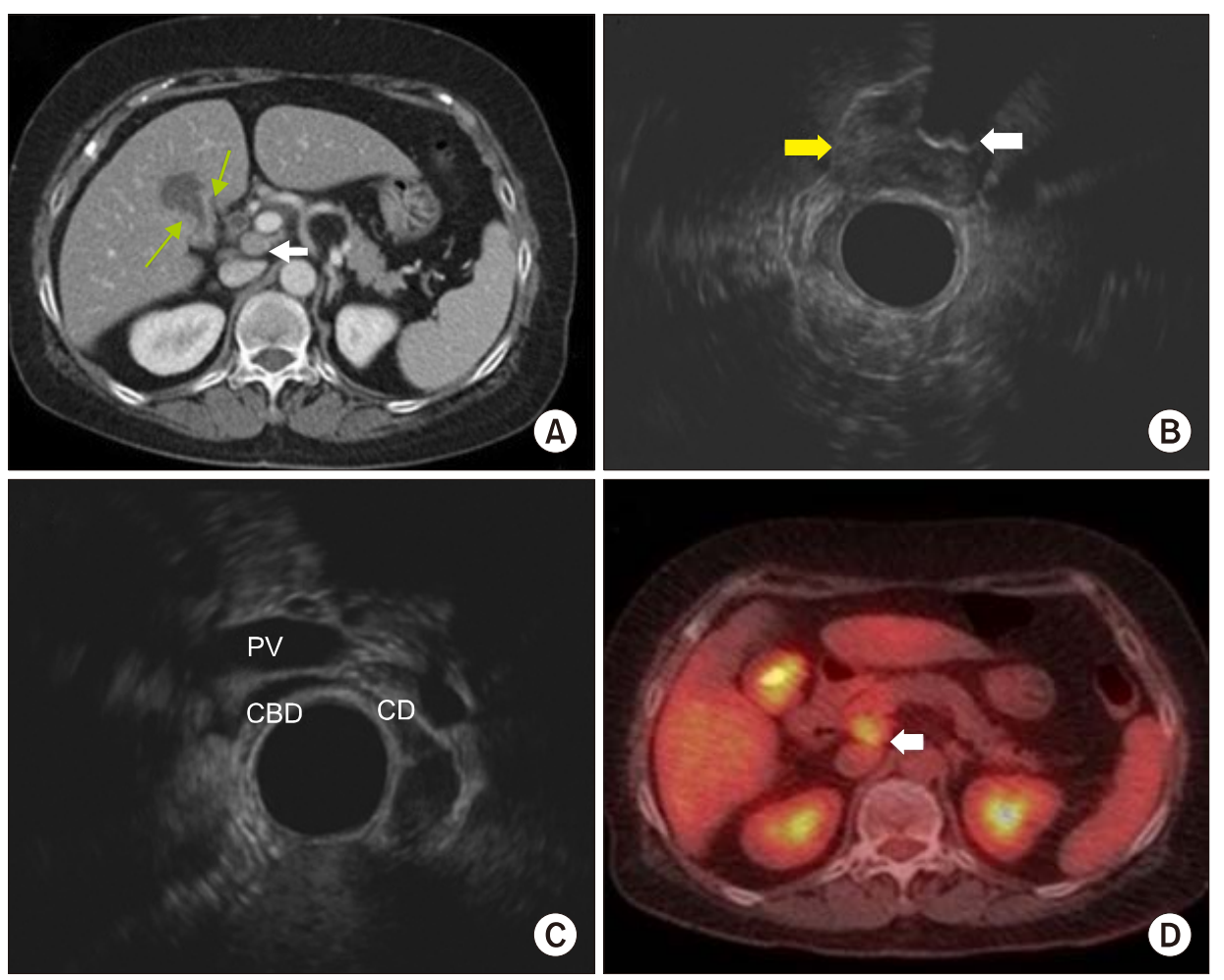

Fig. 1. Preoperative imaging study. (A) Computed tomography scan showing irregular wall thickening of the body to the cystic duct of the gallbladder (green arrow) and portocaval lymph node (white arrow), (B) Endoscopic ultrasound showing intraluminal mass lesion at the neck of the gallbladder without subserosal invasion (yellow arrow) and intraluminal stone (white arrow). (C) Endoscopic ultrasound showing cystic duct involvement of the tumor, (D) Positron emission tomography scan showing intense FDG uptake of in the gallbladder and in the portocaval lymph node (white arrow). 


\section{Operative technique}

Laparoscopic radical cholecystectomy was performed using 6 abdominal ports (Fig. 2). A 12-mm trocar was inserted at the umbilicus, and pneumoperitoneum was established. The patient was then placed in a reverse Trendelenburg position and tilted to the lateral left. Staging laparoscopy was performed. Since there was no sign of distant metastasis, the additional 5 trocars were inserted. Lymphadenectomy was started at the junction of the common hepatic artery and gastroduodenal artery, removing all the lymph nodes and connective tissues surrounding the common hepatic artery and superior margin of the pancreas. Dissection continued along the proper hepatic artery towards the left and right hepatic artery. Since the patient had a tumor involving the cystic duct, the common bile duct resection was performed. The common bile duct was dissected and transected at its most distal extrapancreatic extension. Two $10 \mathrm{~mm}$ hemoclips were applied to secure distal stump. The common bile duct was retracted laterally and superiorly to clearly expose the portal vein. All remaining connective tissues and lymph nodes around the portal vein were dissected up to the confluence of the right and left hepatic ducts. The gallbladder was carefully dissected in a retrograde manner using an electrocautery and was sent for frozen section. It was noted that the tumor invaded the perimuscular connective tissue of the gallbladder and there was no tumor invasion on the liver bed by frozen section. As a result, the liver resection was not performed. The common hepatic duct was resected below the confluence of right and left hepatic duct and the proximal margin was sent for frozen section. There were no tumor cells identified by frozen section.

Next, a full Kocher's maneuver was performed to expose the inferior vena cava and posterior surface of the pancreas. All lymph nodes and connective tissues anterior to the inferior vena cava, retropancreatic, and aortocaval area were removed. Fig. 3A showed the extent of laparoscopic lymphadenectomy. All specimens were collected and placed inside an endopouch.

The hepaticojejunostomy was constructed laparoscopically (Fig. 3B). The jejunum was transected using an endoscopic gastrointestinal anastomosis (GIA) stapling device approximately $50 \mathrm{~cm}$ from the ligament of Treitz. Approximately $50 \mathrm{~cm}$ of retrocolic roux limb was brought up to the common hepatic duct stump and an end-to-side anastomosis was created using a 4-0 vicryl single layer suture (posterior side, continuous running suture; anterior side, simple interrupted suture). Hemostasis was carefully observed and any bleeding vessels were clipped using the endoclip. Two hemovac drains were placed; one in right hepatic area posterior to the hepaticojejunostomy anastomosis, and the other, anterior to the anastomosis.

Finally, the $4 \mathrm{~cm}$ umbilical incision was extended enough

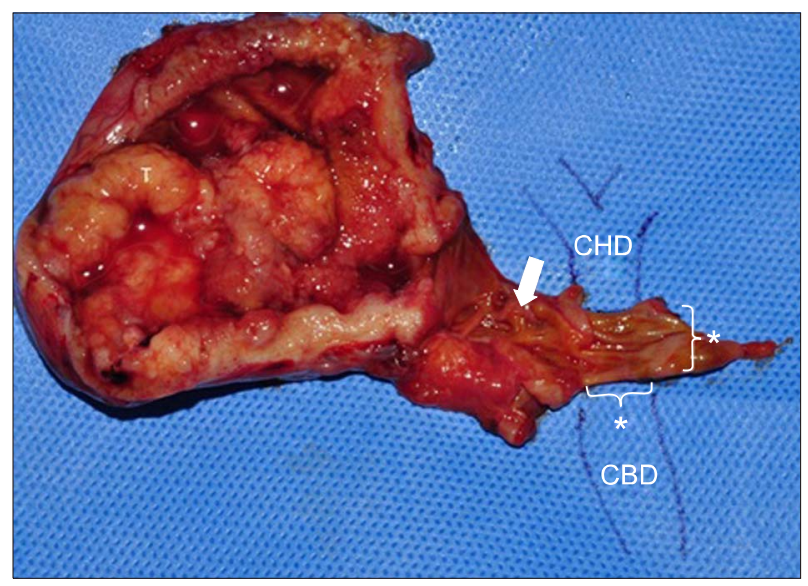

Fig. 4. Specimen image showing gross morphology of the gallbladder tumor and extent of tumor invasion near the cystic duct (white arrow). Note the segment of common bile duct (CBD) $\left(^{*}\right)$.
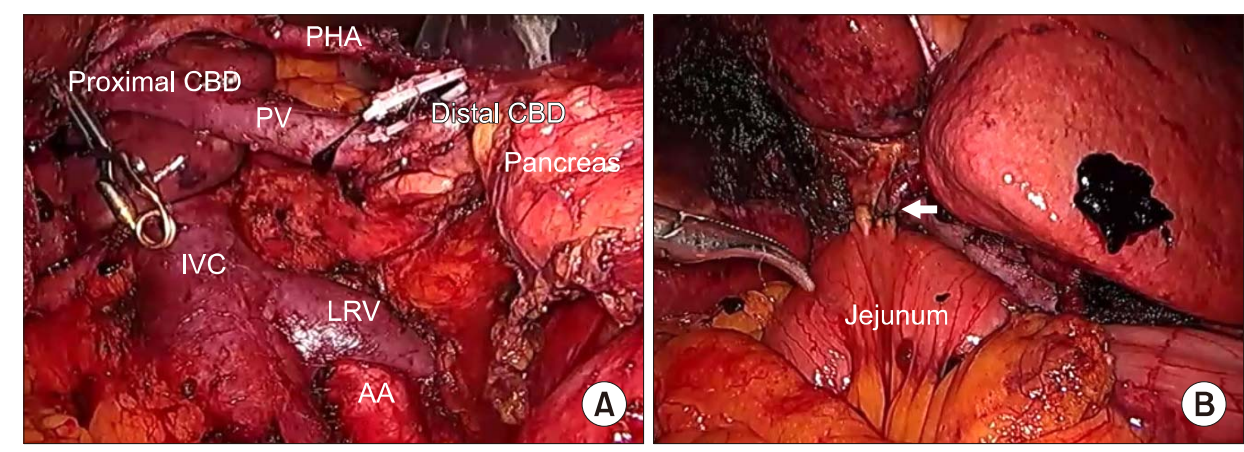

Fig. 3. Intraoperative pictures. (A) Lymph node dissection included station $7,8,9,12,13$, and 16. (B) Completed Roux-en -Y hepaticojejunostomy. PHA, proper hepatic artery; CBD, common bile duct; $\mathrm{PV}$, portal vein; IVC, inferior vena cava; LRV, left renal vein. 
for specimen extraction and extracorporeal jejuno-jejunal anastomosis. A hand sewed side-to-side, double-layer anastomosis was created using vicryl 3-0 sutures.

\section{Pathological findings}

Final pathology report revealed a $4.1 \times 3.0 \mathrm{~cm}$, moderately differentiated adenocarcinoma (Fig. 4). The tumor invades the perimuscular connective tissue on the hepatic side, with no extension into the liver (T2b; American Joint Committee on Cancer $8^{\text {th }}$ Ed.). There were no lymphovascular and perineural invasions. The presence of adenoma, low grade dysplasia, in the cystic duct was noted. A metastatic carcinoma in 1 out of 9 lymph nodes without perinodal soft tissue extension was also reported. The resection margin of the common bile duct was free of tumor.

\section{Post-operative recovery and follow-up}

Patient was started on liquid diet 24 hours postoperatively. Full return of bowel activity was noted on the 3rd postoperative day so patient was placed on full diet. The patient was discharged on the 7th hospital stay with drains removed. Adjuvant chemotherapy with gemcitabine was started at 14 days after surgery and had a regular follow-up check-up in the outpatient department.

\section{DISCUSSION}

The current recommended surgical approach for preoperative suspected T2 gallbladder cancer is open radical cholecystectomy, which includes en bloc liver resection (wedge resection or segment $4 \mathrm{~b} / 5$ resection) and regional lymphadenectomy. Yet, until recently, there is still no consensus as to whether an open approach is superior to laparoscopic surgery. ${ }^{5}$ However, in high volume centers with more experience in minimally invasive surgery, laparoscopic radical cholecystectomy is currently being employed with an oncologic safety and long-term outcome comparable to open approach. ${ }^{6-8}$

In this report, a laparoscopic radical cholecystectomy for preoperatively suspected T2 gallbladder cancer with suspected invasion of the cystic duct was performed. In this approach, an en bloc wedge or segment $4 \mathrm{~b} / 5$ liver resection was not done. For T2 gallbladder cancer, we believe that a simple cholecystectomy with lymph node dissection is adequate as long as intraoperative histologic evaluation confirms a tumor-free liver margin. However, a proper preoperative imaging, including CT scan and EUS, and an intraoperative histopathological evaluation to assess the extent of the tumor invasion is of paramount importance in the decision making whether or not to do extended cholecystectomy. Moreover, in case of liver sided tumor, we usually employed preoperative EUS or intraoperative ultrasound to identify the potential hepatic invasion. As previously reported by Kim et al., ${ }^{9}$ a simple cholecystectomy and lymph node dissection without liver resection had a similar overall survival and recurrence patterns compared with those of radical cholecystectomy in patients with T2 gallbladder cancer. In addition, although hepatic side tumor had a worse long-term outcome compared to peritoneal side tumor, liver resection was not associated with long-term survival. ${ }^{10}$ Yet, even simple cholecystectomy alone has an acceptable oncologic outcome in a properly selected patient with $\mathrm{T} 2$ gallbladder cancer. $^{11}$

Secondly, the extent of lymphadenectomy included the aortocaval lymph nodes (Station 16). Our patient had enlarged lymph nodes at the hepatoduodenal ligament and portocaval space, thus an aortocaval lymph node dissection was performed. The previous study had demonstrated that aortocaval lymph nodes were the common site of tumor recurrence in $\mathrm{T} 2$ gallbladder cancer after radical cholecystectomy. ${ }^{12}$ Moreover, although long-term outcome with aortocaval lymph nodes metastasis is poor and does not benefit from radical resection, ${ }^{13}$ we believe that a proper prognostication can be achieved through extended lymphadenectomy in aortocaval area.

Finally, we employed common bile duct resection to have an adequate tumor-free margin because of the clinically evident tumor invasion to the cystic duct. Our experience in laparoscopic pancreaticoduodenectomy which also includes common bile duct resection and hepaticojejunostomy reconstruction ${ }^{14}$ is one our consideration of adopting the feasibility of minimally invasive approach in gallbladder cancer even with common bile duct invasion. Technically, we employed bile duct to mucosa, end to side hepaticojejunostomy anastomosis with continues running suture on the posterior side and simple interrupted suture on the anterior side, using a vicryl 4-0 suture. With the help of a magnified view during laparoscopy, a healthy and viable tissue of the bile duct can be carefully identi- 
fied and included for the anastomosis. Currently, however, common bile duct resection in gallbladder carcinoma is indicated only if there is evidence of tumor invasion in the cystic duct to achieve an $\mathrm{R} 0$ resection of the primary tumor and if the performance of a complete lymph node dissection that will not be achieved without common bile duct resection. ${ }^{15,16}$

Therefore, with our increasing experience in minimally invasive surgery and mastery in the hepatobiliary anatomy, we are confident that common bile duct resection is not a contraindication for the laparoscopic approach. Nevertheless, this case report is relevant in that it suggests the potential of extending the indication of minimally invasive surgery for gallbladder cancer. Further, extensive lymphadenectomy can be safely and effectively performed laparoscopically especially in high volume center.

It is noteworthy to emphasize the undeniable advantages of the laparoscopic approach over open surgery such as a shorter length of hospital stay, less pain, and early return to work. More importantly, an adjuvant therapy could be started early after the surgery. In certain circumstances when effective systemic chemotherapeutic agents are clinically available in near future, these will be the fundamental advantages of minimally invasive surgery over open surgery.

In summary, this case shows laparoscopic radical cholecystectomy with combined common bile duct resection is technically feasible. This approach should be re-evaluated in terms of reproducibility, and oncologic outcomes based on large number of the patients with long-term follow up data.

\section{REFERENCES}

1. Lee SE, Kim KS, Kim WB, Kim IG, Nah YW, Ryu DH, et al. Practical guidelines for the surgical treatment of gallbladder cancer. J Korean Med Sci 2014;29:1333-1340.

2. Aloia TA, Járufe N, Javle M, Maithel SK, Roa JC, Adsay V, et al. Gallbladder cancer: expert consensus statement. HPB (Oxford) 2015;17:681-690.

3. Agarwal AK, Javed A, Kalayarasan R, Sakhuja P. Minimally invasive versus the conventional open surgical approach of a radical cholecystectomy for gallbladder cancer: a retrospective comparative study. HPB (Oxford) 2015;17:536-541.

4. Gumbs AA, Hoffman JP. Laparoscopic radical cholecystectomy and Roux-en-Y choledochojejunostomy for gallbladder cancer. Surg Endosc 2010;24:1766-1768.

5. Yoon YS, Han HS, Agarwal A, Belli G, Itano O, Gumbs AA, et al. Survey results of the expert meeting on laparoscopic surgery for gallbladder cancer and a review of relevant literature. Dig Surg 2019;36:7-12.

6. Yoon YS, Han HS, Cho JY, Choi Y, Lee W, Jang JY, et al. Is laparoscopy contraindicated for gallbladder cancer? A 10-Year Prospective Cohort Study. J Am Coll Surg 2015;221:847-853.

7. Zhang L, Hou C, Xu Z, Wang L, Ling X, Xiu D. Laparoscopic treatment for suspected gallbladder cancer confined to the wall: a 10-year study from a single institution. Chin J Cancer Res 2018;30:84-92.

8. Zimmitti G, Manzoni A, Guerini F, Ramera M, Bertocchi P, Aroldi F, et al. Current role of minimally invasive radical cholecystectomy for gallbladder cancer. Gastroenterol Res Pract 2016;2016:7684915.

9. Kim DH, Kim SH, Choi GH, Kang CM, Kim KS, Choi JS, et al. Role of cholecystectomy and lymph node dissection in patients with T2 gallbladder cancer. World J Surg 2013;37:2635-2640.

10. Park TJ, Ahn KS, Kim YH, Kim TS, Hong JH, Kang KJ. The optimal surgical resection approach for T2 gallbladder carcinoma: evaluating the role of surgical extent according to the tumor location. Ann Surg Treat Res 2018;94:135-141.

11. Kang CM, Lee WJ, Choi GH, Kim JY, Kim KS, Choi JS, et al. Does "clinical" R0 have validity in the choice of simple cholecystectomy for gallbladder carcinoma? J Gastrointest Surg 2007;11:1309-1316.

12. Park JS, Yoon DS, Kim KS, Choi JS, Lee WJ, Chi HS, et al. Actual recurrence patterns and risk factors influencing recurrence after curative resection with stage II gallbladder carcinoma. J Gastrointest Surg 2007;11:631-637.

13. Meng H, Wang X, Fong Y, Wang ZH, Wang Y, Zhang ZT. Outcomes of radical surgery for gallbladder cancer patients with lymphatic metastases. Jpn J Clin Oncol 2011;41:992-998.

14. Rho SY, Kim JS, Chong JU, Hwang HK, Yoon DS, Lee WJ, et al. Indocyanine green perfusion imaging-guided laparoscopic pancreaticoduodenectomy: potential application in retroperitoneal margin dissection. J Gastrointest Surg 2018;22:1470-1474.

15. Shukla PJ, Barreto SG. Systematic review: should routine resection of the extra-hepatic bile duct be performed in gallbladder cancer? Saudi J Gastroenterol 2010;16:161-167.

16. Pandey D, Garg PK, Manjunath NM, Sharma J. Extra-hepatic bile duct resection: an insight in the management of gallbladder cancer. J Gastrointest Cancer 2015;46:291-296. 